\title{
Des loups en peau humaine : Faux Semblant et les appétits animaux dans Le Roman de la rose de Jean de Meun
}

Jonathan Morton

\section{(2) OpenEdition}

\section{Journals}

Édition électronique

URL : http://journals.openedition.org/questes/107

DOI : 10.4000/questes. 107

ISSN : 2109-9472

Éditeur

Les Amis de Questes

\section{Édition imprimée}

Date de publication : 15 avril 2013

Pagination : $99-119$

ISSN : 2102-7188

\section{Référence électronique}

Jonathan Morton, «Des loups en peau humaine : Faux Semblant et les appétits animaux dans Le Roman de la rose de Jean de Meun », Questes [En ligne], 25 | 2013, mis en ligne le 01 janvier 2014, consulté le 19 avril 2019. URL : http://journals.openedition.org/questes/107 ; DOI : 10.4000/ questes.107 


\title{
Des loups en peau humaine : Faux Semblant et les appétits animaux dans Le Roman de la Rose de Jean de Meun
}

\author{
Jonathan MORTON \\ Université d'Oxford (New College)
}

Je ne l'oi pas plus tost passee

Qu'Amors trouvai dedanz la porte, et son ost qui confort m'aporte.

Dex! quel avantaige me firent

li vassal qui la desconfirent !

De Dieu et de saint Benoait

puissent il estre benoait !

Ce fu Faus Samblant, li traïstres, le filz Barat, li faus ministres deYpocrisie sa mere, qui tant est au vertuz amere, et dame Attenance Contrainte, qui de Faus Samblant est enceinte, preste d'anfanter Antecrit, si con je truis an livre ecrit.

Ne sunt religieus ne monde ;

il font un argument au monde ou conclusion a honteuse :

cist a robe religieuse, donques est il religieus.

Cist argumenz est touz fieus, il ne vaut pas un coustel troine : la robe ne fet pas le moine. ${ }^{1}$

Dans la première partie du Roman de la rose de Guillaume de Lorris, l'allégorie est un vêtement gracieux et civilisateur qui couvre les vérités malséantes du désir sexuel de l'Amant. Les arts poétiques de la fin du $\mathrm{XII}^{\mathrm{e}}$ siècle et du $\mathrm{XIII}^{\mathrm{e}}$ siècle considèrent en effet que l'integumentum

\footnotetext{
${ }^{1}$ Guillaume DE LoRris et Jean DE Meun, Le Roman de la rose, Félix LeCoy (éd.), Paris, Champion, CFMA, 3 vol., 1965-1970, t. 2, v. 11021-11028.
} 
dissimule la vérité nue sub pio figmentorum velamine ${ }^{2}$, "sous un voile pudique d'inventions », c'est-à-dire sous un langage dont la modestie a une valeur équivalente à celle des vêtements comme signe de civilisation et d'humanité ${ }^{3}$. Les conventions du récit courtois et l'emploi d'une allégorie qui a valeur d'euphémisme dissimulent et maîtrisent ce désir tout en en laissant filtrer une évocation édulcorée et acceptable. Bien qu'il soit indiscutable que le personnage central du texte de Guillaume désire une femme, l'objet de désir est représenté métaphoriquement comme une rose qu'il veut embrasser, puis « cueillir », c'est-à-dire déflorer. La sexualité est sublimée dans le but de ne pas transgresser les règles sociales de la conduite courtoise. Jean de Meun, dans sa continuation du poème, met à nu ces voiles protecteurs de la civilisation pour exposer la véritable nature, animale, des appétits humains. Ce processus provoque une réévaluation du rôle que jouent les pulsions physiques dans la psyché humaine, et révèle le caractère poreux de ces enveloppes sous le couvert desquelles nous cherchons à contrôler et masquer l'animal présent dans l'homme. Cet article portera sur le motif des êtres humains transformés

\footnotetext{
${ }^{2}$ Ambrosius Aurelius Theodosius Macrobius (MACROBE), Commentaire au Songe de Scipion, Mireille ARMisen Marchetti (éd. et trad.), Paris, Les Belles Lettres, "Collection des Universités de France. Série latine», 2 vol., 2003, t. I, 2, 11. Voir également Geoffroy De Vinsauf, Poetria nova, Martin CAMARgo (éd.) et Margaret F. Nims (trad.), Toronto, Pontifical Institute of Medieval Studies, « Medieval Sources in Translation », 2010, t. I, 60-70. Pour une discussion plus approfondie de la relation entre vêtements et théorie littéraire au Moyen Âge, voir Mary Frances Brown, «Critique and Complicity : Metapoetical Reflections on the Gendered Figures of Body and Text in the Roman de la rose», Exemplaria, Binghampton, New York, Medieval and Renaissance Texts and Studies, 21, 2 (2009), p. 129-159.

${ }^{3}$ Albert le Grand, pour déterminer si les Pygmées doivent compter au nombre des animaux ou des humains s'appuie sur la nature de leur langage : il indique selon lui un manque de raison et d'intelligence «propter quod etiam rhetoricis persuasionibus in loquendo non utitur neque poeticis » (« car ils ne font usage, dans leurs discours, ni des procédés rhétoriques, ni des procédés poétiques. », nous traduisons). ALBERTUS Magnus, De animalibus libri XXVI, Hermann StAdLer (éd.), Münster, Aschendorffsche Verlagsbuchhandlung, « Beiträge zur Geischichte der Philosophie des Mittelalters », 2 vol., 1920-1916, t. II, XXI. 1. 2. 13. L'emploi de la rhétorique, et plus encore de la raison théorique, forment aux yeux d'Albert une caractéristique propre à l'être humain.
} 
métaphoriquement en animaux ou, plutôt, sur le motif des animaux surtout des loups - qui prennent une apparence humaine pour mieux chasser leur proie, humaine elle-aussi : cette image se cristallise autour du personnage trouble de Faux Semblant.

Faux Semblant, «l'ort hypocrite au queur porri, / qui traït mainte region / par habit de religion $»^{4}$, se présente au Dieu d'Amour et à ses barons en ces termes :

Je suis des vallez Antecrit, des larrons don il est escrit qu'il ont habit de saintée et vivent en tel faintée.

Dehors semblons aigneaus pitables, dedanz somes lous ravisables. ${ }^{5}$

Le public fictionnel de Faux Semblant et le public réel de Jean de Meun doivent faire face à une incertitude troublante et déstabilisante concernant notre capacité à juger de la vérité d'autrui. Dans le poème de Guillaume de Lorris, il n'y a guère de distinction entre l'apparence d'un personnage allégorique et sa vraie nature. Par contraste, Faux Semblant brise ce lien : son apparence, nous dit-il, n'est qu'un mensonge, total et diabolique. L'introduction de l'Antéchrist dans le verger de Déduit, comme l'apparition du péché dans le jardin d'Éden, marque un éloignement du monde allégorique harmonieux et civilisé de Guillaume. La nature même de Faux Semblant, hypocrite archétypique, nous met dans l'incapacité de nous fier au moindre mot issu de sa bouche, et l'hypocrisie, qui caractérise l'homme en propre, dans la mesure où elle ne se trouve chez aucun autre animal, rend problématique la connaissance de la «vraie» nature de l'autre. Le moment même où apparaît Faux Semblant, qui correspond au milieu du roman, souligne le caractère central de ce doute chez Jean

\footnotetext{
${ }^{4}$ Guillaume DE LORRIS et Jean DE MEUn, Le Roman de la rose, éd. cit., v. 10442-10444.

${ }^{5}$ Ibid., v. 11683-11688.
} 
de Meun ${ }^{6}$. Ce qui est frappant c'est que cette problématisation de la communication humaine rejoint la menace que, sous des vêtements et des paroles raffinés, l'homme ne soit que trop semblable aux animaux. Cette similitude n'apparente pas l'homme à n'importe quelles bêtes, mais aux loups cruels et rapaces, aux «lous ravisables ». Non seulement l'homme peut être malfaisant, mais, en un sens, il peut cesser d'être humain. Jean de Meun prend appui sur la tradition poétique qui met en scène des animaux, surtout des loups, qui se comportent comme des humains pour explorer l'animalité qui se cache au-dedans de nous, une animalité qui peut surgir à n'importe quel moment pour mettre à mal certains aspects de la position privilégiée que l'homme se vante d'occuper comme espèce supérieure.

\section{Le loup déguisé en moine : rapacité et hypocrisie}

\section{La rapacité : le loup dévoreur de moutons}

Faux Semblant se déguise en religieux en affirmant que l'apparence de la pureté rend plus aisée la mise en œuvre de crimes qui ont pour but de satisfaire les pulsions de domination et de possession matérielle et sexuelle. C'est cette identification avec l'avarice et la satisfaction des désirs qui le pousse à se faire - métaphoriquement - loup. La signification symbolique du loup dans la culture de l'Occident médiéval, son association avec la cruauté et l'appétit et ses liens avec l'hypocrisie, donnent tout son poids au choix de cette image pour représenter le religieux hypocrite, ou, pour être plus précis, pour représenter l'hypocrisie-en-vêtements-de-religieux. Les attitudes médiévales à l'égard du loup peuvent en premier lieu être examinées à travers les bestiaires, en l'occurrence celui du manuscrit 24 de

\footnotetext{
${ }^{6}$ Pour un traitement détaillé de la position de Faux Semblant et de la tromperie dans Le Roman de la rose, voir Susan STAKEL, False Roses : Structures of Duality and Deceit in Jean de Meun's "Roman de la Rose », Saratoga, Anma Libri, "Stanford French and Italian Studies », 1991.
} 
la bibliothèque universitaire d'Aberdeen ${ }^{7}$. L'article sur le loup commence, de manière caractéristique, par l'étymologie du nom de l'animal :

Lupus greca dirivatione in linguam nostram transfertur. Lupos enim dicunt illi licos, licos autem grece, a morsibus apellantur, quod rabie rapacitatis, queque invenerint trucidant. $^{8}$

Le loup devient un symbole du vice vorace et cruel, motivé par son appétit: la bête par excellence. Cette représentation reçoit l'appui de la Bible et de son autorité. Dans la Genèse ${ }^{9}$, Jacob bénit Benjamin ainsi : «Beniamin lupus rapax; mane comedat praedam et uespere diuidet spolia ${ }^{10}$.» Le loup est la figure biblique de la rapacité. Dans d'autres passages de la Bible $^{11}$, le loup renvoie aux dirigeants sans pitié qui dépouillent leurs sujets. La permanence de cette image traditionnelle du loup au Moyen Âge est attestée par une citation de Grégoire le Grand dans la Summa theologiae de Thomas d'Aquin: «Dicit enim Gregorius, in quadam homilia, lupus super oues uenit, cum quilibet iniustus et raptor fideles quosque atque humiles opprimit $»^{12}$. Faux Semblant reprend lui aussi cette idée dans sa description des puissants : « Li menuz pueple les encline, / Et cil conme lou les deveurent ${ }^{13}$. L'emploi par Grégoire et

\footnotetext{
${ }^{7}$ Nous suivons dans ce choix Alexander Plukowski, qui l'utilise comme exemple typique. Aleksander Pluskowski, Wolves and the Wilderness in the Middle Ages, Woodbridge, Boydell, 2006, p. 129.

${ }^{8}$ «Lupus a été transposé dans notre langue à partir du grec, car les Grecs appellent les loups licos; ils sont appelés ainsi à cause de leurs morsures par lesquelles ils massacrent tout ce qu'ils trouvent dans leur rapacité furieuse » (Aberdeen, Aberdeen University Library, ms. 24, $\mathrm{f}^{\circ} 16 \mathrm{v}^{\circ}$, nous traduisons).

${ }^{9}$ Genèse, XLIX, 27. Les citations bibliques sont tirées de la Vulgate de Saint Jérôme, dans l'édition en ligne de la Biblia Sacra : http://www.thelatinlibrary.com/bible.html

${ }^{10}$ «Benjamin est semblable à un loup ravisseur. De grand matin, il dévore sa proie, et sur le soir encore, il partage le butin » (nous traduisons).

${ }^{11}$ Ézéchiel, XXII, 27 et Sophonie, III, 3.

12 «Car Grégoire dit dans une de ses homélies, "le loup vient sur les moutons chaque fois qu'un pilleur injuste opprime les fidèles et les humbles". » (THOMAS D'AQUIN, Summa theologiae, Roberto BuSA (éd.), Stuttgart, Fromman-Holzboog, 1980, IIa-IIae. q. 40 , a. 2 ag1, nous traduisons).

13 Guillaume De Lorris et JEAn DE MeUn, Le Roman de la rose, éd. cit., v. 11512-11513.
} 
Thomas d'une opposition entre le loup et le mouton se rattache à la tradition du Nouveau Testament, qui donne des loups une vision un peu différente, et suit la présentation du loup comme l'ennemi naturel des moutons, récurrente chez Esaï ${ }^{14}$. Cette tradition rejoint au Moyen Âge l'expérience vécue des loups prédateurs qui dévorent le bétail, pour donner naissance à une opposition durable entre le mouton ou l'agneau innocents et le loup rapace et démoniaque. Il faut pourtant souligner que, avant le sermon de Genius qui se trouve vers la fin de l'ouvrage, Jean de Meun ne représente pas les moutons dans le cadre chrétien usuel, comme les disciples de Jésus, mais plutôt au sein d'une lutte proto-darwinienne où ils sont les animaux faibles et destinés à être mangés. Même dans l'allégorie christique de Genius, la conception de l'existence humaine comme combat animal persiste: les brebis guidées par l'Agneau sont des victimes, poursuivies, et éventuellement dévorées, par le diable, qui est un « lous ${ }^{15}$. La valeur spirituelle et le salut sont bien éloignés de ce traitement des activités bestiales des hommes.

\section{L'hypocrisie : le loup déguisé en mouton et la satire des religieux}

Le religieux, celui qui renonce au désir charnel pour mener une existence plus sainte, est le mouton chrétien archétypique, vrai disciple de l'Agneau de Dieu. Aussi l'association des loups aux religieux est-elle naturelle pour les satiristes qui veulent critiquer les hypocrisies charnelles de l'existence monacale. Ils peuvent s'appuyer sur un passage de Matthieu : «Attendite a falsis prophetis, qui ueniunt ad vos in uestimentis ouium, intrinsecus autem sunt lupi rapaces $»^{16}$.

\footnotetext{
${ }^{14}$ Voir Esaïe, XI, 6 et LXV, 25.

${ }^{15}$ GuIllaume de LorRis et JEAN DE Meun, Le Roman de la rose, éd. cit., v. 20230.

16 «Gardez-vous des faux prophètes! Lorsqu'ils vous abordent, ils se donnent l'apparence d'agneaux mais, en réalité, ce sont des loups féroces » (Matthieu, VII, 15, nous traduisons).
} 
Dans le De lupo, par exemple, poème satirique du $\mathrm{XI}^{\mathrm{e}}$ siècle, un homme feint une conversion pour pouvoir porter une tonsure et un habit de moine qui lui permettent de mieux tromper un berger et de lui voler ses moutons ${ }^{17}$. Le motif du loup-en-moine voit son traitement le plus soutenu dans le poème latin anonyme Ysengrimus, écrit en Flandres au milieu du XII ${ }^{\mathrm{e}}$ siècle et dont l'intrigue principale est la vendetta qui oppose Ysengrimus, le loup, à Reinardus son neveu, le renard ${ }^{18}$. Dans ce texte, c'est pour leur avarice que les moines sont condamnés. Ils se précipitent avec la violence de la foudre sur toute occasion de s'enrichir : « Monachus oblatu cum uideritat fore lucrum, / irruit ut pluuio fulge tra mota polo $»^{19}$. L'appétit du moine dépasse même celui du loup: Ysengrimus remarque que « Dico satur "satis est", monachususque "parum est" »". Il y a là un effet de symétrie lié au renversement ironique de la pureté hyperbolique à laquelle les moines sont normalement associés comme brebis chrétiennes : sous l'effet de l'inversion ${ }^{21}$, elle se transforme en bestialité hyperbolique.

Avant de poursuivre l'étude et de nous pencher sur les implications théoriques de cette union satirique des natures humaine et animale, un dernier exemple montrera le décalage qui résulte de la confusion entre bête rapace et humain rationnel, à savoir le motif du «loup à l'école», qui est

17 De lupo, Friedrich Walter LENZ (éd.), «Bemerkungenzudem pseudo-ovidischen Gedicht De lupo », Orpheus, 10 (1963), p. 21-32 et p. 25-28. Voir Jan M. ZIOLKOWSKI, Talking Animals : Medieval Latin Beast Poetry, 750-1150, Philadelphie, University of Pennsylvania Press, «Middle Ages Series », 1993, p. 202 et p. 301-303. Ziolkowski fournit une chronologie utile des sources médiévales où les loups se déguisent en moines, p. 202-210.

${ }^{18} \mathrm{Il}$ n'est pas très difficile de retrouver les incarnations françaises de ces personnages au XIII ${ }^{\mathrm{e}}$ siècle dans les contes du Roman de Renart.

19 «Quand un moine voit l'occasion de gagner de l'argent, il tombe là-dessus comme l'éclair de foudre que produit un ciel orageux » (Ysengrimus, Jill MANN (éd.), Leyde, Brill, « Mittellateinische Studien und Texte », 1987, v. 639-640, nous traduisons).

${ }^{20}$ "Quand je suis rassasié, je dis "ça suffit", alors que le moine dit "c'est peu" » (Ibid., v. 644 , nous traduisons).

${ }^{21}$ Cette inversion est à la fois celle à laquelle procède l'ironie (du point de vue des procédés rhétoriques mis en œuvre par la satire), et celle que l'ironie entend dénoncer dans la réalité des pratiques. 
largement répandu aussi bien dans les différentes formes d'art que dans les littératures latine, allemande, française et anglaise ${ }^{22}$. Dans toutes les versions de ce conte, un clerc ou un moine s'efforce d'enseigner au loup soit l'alphabet soit le credo, et la bête ne peut pas s'empêcher de penser aux moutons. Une bulle papale d'Urbain II du 14 avril 1096 destinée aux moines du monastère de Montieurneuf au sujet des demandes adressées aux moines par les clercs séculiers, démontre la banalité du thème du Loup à l'école :

Nos uero anima duertentes, non eos pro spiritualibus causari, sed pro carnalibus, serio diximus quoddam prouerbium, quod debuerat eis uerecundiam inferre, si aduertere uoluissent, de lupo ad discendas litteras posito, cui cum magister diceret A, ipse agnellum, et cum magister B, ipse dicebat porcellum. Sic et ipsi faciebant, quia, cum nos promitteremus psalmos et orationes, ipsie contra quere bantea, quae non sunt ad profectum animarum proficua. ${ }^{23}$

Le loup monacal, ou plutôt le moine lupin, est un personnage bien connu en Europe médiévale : il représente la prise en compte de la part charnelle qui existe chez tous les êtres humains, y compris chez ceux qui sont censés être les plus saints. Les moines, dans l'Ysengrimus, ne sont pas comme des loups, mais effectivement ils sont des loups, puisqu'un loup peut se faire passer pour un moine sans avoir à accomplir d'autres gestes que se faire tonsurer et revêtir des habits monacaux, les uestimentum ovis, comme le dit

${ }^{22}$ Jan M. ZIOLKOWSKI, Talking Animals..., op. cit., p. 206-208. Pour un traitement plus développé voir également Ayers BAGLEy, «A Wolf at School», Studies in Medieval and Renaissance Teaching, Wichita, Kansas, Fairmount College of Liberal Arts and Sciences, Wichita State University, 4, 2 (1993), p. 35-69.

${ }^{23}$ «En vérité, nous étant rendus compte qu'ils plaidaient non pas pour des privilèges spirituels mais pour des privilèges charnels, nous avons sérieusement dit un proverbe qui devait leur faire honte, s'ils voulaient en tenir compte, celui du loup assis pour apprendre son alphabet, qui, quand le maître disait "A", répondait "agneaux" et quand le maître disait "B", répondait, "porcelet". Ils faisaient pareil, parce que quand nous avons promis des psaumes et des prières, leur réponse était de demander des choses qui ne sont pas bénéfiques pour le profit des âmes. " Bulle papale citée dans Kleinere lateinische Denkmäler der Thiersage aus dem zwölften bis vierzehnten Jahrhundert, Ernst VoIGT (éd.), Strasbourg, Trübner, «Quellen und Forschungen zur Sprach- und Culturgeschichte der germanischen Völker »,1878, p. 21, nous traduisons. 
Jean-Yves Tilliette en reprenant une expression de Matthieu, VII, $15 .{ }^{24} \mathrm{La}$ première référence aux loups dans le Roman de la rose est faite par Faux Semblant qui parle de son déguisement en moine. Il se justifie en disant que

Qui de la toison dam Belin au leu de mantel sebelin sire Isengrin affubleroit, li lous, qui mouton sembleroit, por qu'o les berbiz demorast, cuidiez vos qu'il nes devorast ? $^{25}$

Faux Semblant se présente donc bel et bien comme l'héritier de cette tradition satirique anti-religieuse ${ }^{26}$.

\section{L'association à l'antéchrist et la portée polémique du loup en peau de mouton}

\section{La Querelle de l'Université et le thème apocalyptique}

Nous voudrions revenir à présent sur l'affirmation de Faux Semblant, qui se présente comme « des vallez Antecrit », pour mieux considérer une autre branche de cette tradition du loup-religieux et l'usage qu'en fait Jean

\footnotetext{
${ }^{24}$ Jean-Yves Tilliette, « La peau de loup, l'apocalypse : remarques sur le sens et la construction d'Ysengrimus ", Médiévales, Paris, Presses Universitaires de Vincennes, 38 (2000), p. 163-176, cit. p. 168.

25 Guillaume De LORRIS et JEAN DE MEUn, Le Roman de la rose, éd. cit., v. 11093-11098. «Belin » ou « Belinus » est le nom du bélier dans le Roman de Renart et dans Ysengrimus, bien que cet épisode de déguisement ne figure dans aucun des deux textes.

${ }^{26}$ Le motif du déguisement à l'aide d'une peau de brebis n'apparaît pas tel quel dans l'Ysengrimus ou le Roman de Renart. D'après Andrea Valentini, cette ruse particulière pourrait être empruntée à Eude de Chériton, qui la fait utiliser par le renard (Andrea VALENTINI, "Le remaniement de Gui de Mori et sa tradition manuscrite », dans Catherine BEL et Herman BRAET (dir.), De la Rose : texte, image, fortune, Louvain, Peeters, «Synthema », 2006, p. 299-230). Andrea Valentini observe néanmoins que chez Eudes, loup et renard sont souvent interchangeables. L'emploi du nom « dan Belin » pour désigner le mouton implique par ailleurs une fusion de la fable d'Eude avec l'univers de l'Ysengrimus et du Roman de Renart. L'allusion de Jean à Ysengrin accentue donc l'association du loup à un moine immoral et pose les bases d'allusions ultérieures au loup comme symbole d'appétit et de tromperie.
} 
de Meun. Comme on l'a vu, ce vers renvoie à un passage de Matthieu ${ }^{27}$, et aux faux prophètes qui sont comparés à des loups en peaux de moutons, mais la mention de l'Antéchrist lie Faux Semblant aux polémiques antireligieuses qui ont lieu à l'Université de Paris dans la deuxième moitié $\mathrm{du} \mathrm{XIII}^{\mathrm{e}}$ siècle, à l'époque où Jean de Meun écrit sa continuation du Roman de la rose. En dépit du contexte de ce conflit entre les maîtres ès arts séculiers et les frères, Guy Geltner soutient qu'on ne doit pas comprendre le personnage de Faux Semblant comme une satire des frères mais comme une représentation de l'hypocrisie tout court, en prenant pour argent comptant l'affirmation de Faux Semblant selon laquelle il est «a personification of hypocrisy, not a direct depiction or a caricature or a real (or aggregate) friar $»^{28}$. Pourtant, Geltner reconnaît que Faux Semblant propose à Amour et au lecteur le paradoxe classique du menteur crétois on ne peut se fier aux paroles de qui se dit menteur, pas même à cette affirmation par laquelle il se reconnaît menteur, et l'on ne peut par conséquent prendre aucun mot pour argent comptant. Pour lui, la critique des frères repose uniquement sur l'éloge que fait Faux Semblant des œuvres composées par Guillaume de Saint-Amour contre les ordres mendiants, dans les années 1250, et sur le fait que le nom «Faux Semblant» est celui utilisé par Rutebeuf pour l'un des personnages de l'entourage d'Hypocrisie dans La Complainte Maitre Guillaume de Saint Amour, un poème qui défend Guillaume ${ }^{29}$. Cependant, comme on l'a déjà

\footnotetext{
${ }^{27}$ Matthieu, VII, 15.

${ }^{28}$ « une incarnation de l'hypocrisie, et non une représentation directe, une caricature ou un frère réel (ou composé à partir de plusieurs ordres)» (Guy GELTNER, "Faux Semblants : Antifraternalism Reconsidered in Jean de Meun and Chaucer », Studies in Philology, Chapel Hill, North Carolina, University of North Carolina Press, 101, 4 (2004), p. 357-80, cit. p. 359, nous traduisons ). Voir également Daniel POIRION, « Jean de Meun et la querelle de l'Université de Paris : du libelle au livre », dans Traditions Polémiques, Paris, École Normale Supérieure des Jeunes Filles, «Collection de l'École Normale Supérieure de Jeunes Filles », 27, 1984, p. 9-19.

${ }^{29}$ Rutebeuf, Euvres complètes de Rutebeuf, Edmond Faral et Julia BASTin (éd.), Paris, Picard, 2 vol., 1969, t. I, p. 258-266.
} 
vu, l'image du loup dont se sert Jean de Meun est héritée d'une tradition satirique tournée contre l'hypocrisie des religieux. Quoi qu'en dise Faux Semblant, les métaphores lupines du texte témoignent d'une attaque voilée contre les frères et les religieux en général, attaque qui se dissimule derrière le voile que constitue la possibilité pour le poète de présenter sa satire comme une image générale de l'hypocrisie, et donc de nier la spécificité de sa cible. Jean de Meun marche dans les pas de Guillaume de Saint-Amour, tant par sa tentative pour se dédouaner des accusations de polémique antireligieuse que par l'emploi d'images apocalyptiques. Penn Szittya a remarqué que dans tous les ouvrages de Guillaume condamnés comme anticléricaux, dont le plus notoire est son Tractatus de periculis nouissimorum temporum ex Scripturis sumptus, « the most startling feature $[\ldots]$ is the almost total absence of any reference to the friars themselves $\gg{ }^{30}$. Ses textes prétendent en effet - non sans un certain manque de sincérité ne viser aucun des ordres de l'Église et n'être que des exégèses de passages bibliques qui exposent les périls des temps derniers prophétisés par l'Écriture. Des échos de cette technique se retrouvent chez Rutebeuf, qui rehausse sa critique des frères à l'aide d'allusions à des versets bibliques qui traitent de l'hypocrisie. C'est le cas, par exemple, dans son $D u$ Pharisien, un poème qui, écrit à la suite d'un sermon polémique de Guillaume de Saint-Amour, ferait allusion à Matthieu, VII, 15 (verset que cite souvent Guillaume lui-même ${ }^{31}$ ) et au passage célèbre de Matthieu XXIII, 2-8 dirigé contre 1'hypocrisie des Pharisiens ${ }^{32}$. Le Faux Semblant de Jean de Meun rappelle celui qui accompagnait l'Hypocrisie de Rutebeuf, et reprend le motif de l'apocalypse quand il se dit « des vallez Antecrist », ce

\footnotetext{
${ }^{30}$ «L'aspect le plus frappant [...] est l'absence presque totale de toute référence aux frères eux-mêmes. " (Penn R. SzITTYA, The Antifraternal Tradition in Medieval Literature, Princeton, Princeton University Press, 1986, p. 18, nous traduisons).

${ }^{31}$ Rutebeuf, Euvres complètes de Rutebeuf, éd. cit., t. I, p. 252, v. 51 ; voir SzITTYA, The Antifraternal Tradition in Medieval Literature, op. cit., p. 184-185.

${ }^{32}$ Faux Semblant fait lui aussi référence à Matthieu, XXIII, 2-8 (vers 11576-11600).
} 
qui fait référence à Jean, II, 18: «filioli, nouissima hora est; et sicut audistis quia Antichristus uenit nunc Antichristi multi facti sunt: unde scimus quoniuam nouissima hora est ${ }^{33}$. L'association de la figure de l'Antéchrist et, par là même, de l'Apocalypse, à l'image des loups en vêtements de mouton, renforce, à travers ces effets intertextuels, l'inscription de Faux Semblant dans la lignée des ouvrages contre les ordres mendiants de Guillaume de Saint-Amour (et dans la tradition plus large de la satire anticléricale), et ce en dépit des protestations du personnage lui-même, qui se défend de critiquer l'ensemble des religieux : «Si ne vueill je mie blamer / religion ne diffamer / en quel que habit que l'en la truisse ${ }^{34}$. Faux Semblant, le loup rapace, s'assimile seulement à ceux des ordres religieux qui n'ont pas rejeté la vie charnelle ou l'avarice, et sont visés précisément à cause de cela. Cela relève d'une critique spécifique des religieux qui ont pris les vetements et l'apparence d'une soidisant pureté, tout en renvoyant en même temps à une satire plus générale de l'hypocrisie, quel que soit l'état où elle se manifeste. En effet, les ordres religieux sont les premiers et les principaux exemples d'êtres humains qui prétendent supprimer leurs appétits animaux mais se révèlent aussi rapaces, aussi bestiaux que les autres, sinon plus.

\section{Le religieux, un cas exemplaire représentatif de l'ensemble de la société}

Le moine ou le frère bestial devient alors le symbole archétypique de l'échec plus général des institutions humaines à freiner nos pulsions animales de domination et notre concupiscence. Geltner a raison de suggérer que Faux Semblant ne vise pas seulement les religieux; le

\footnotetext{
33 "Mes enfants, la dernière heure a commencé. Vous avez appris qu'un " anti-Christ” doit venir. Or, dès à présent, beaucoup d'“antichrists" sont là. Voilà pourquoi nous savons que nous sommes entrés dans la dernière heure » (nous traduisons).

34 Guillaume De LoRris et JeAn DE Meun, Le Roman de la rose, éd. cit., v. 10987-10989.
} 
personnage affirme explicitement que les personnes dans lesquelles il loge se comportent toutes de la même manière « quelconques robes que il aient, / de quelconques estat qu'il soient ${ }^{35}$. Les habits, l'apparence extérieure ne sont pas forcément liés à l'état intérieur. Faux Semblant développe sa thèse peu après : «Bon queur fet la pensee bone, / la robe n'i tost ne ne done » ${ }^{36}$. Cette affirmation s'inscrit dans un passage où il rejette le besoin de porter les habits d'un ordre religieux pour être saint, en prenant comme exemple les vies des nombreux saints qui étaient laïques et non pas religieux ${ }^{37}$. Les conventions extérieures, les extérieurs conventionnels - ici les vêtements ne donnent aucun indice fiable sur le vrai caractère d'autrui et ne le rendent pas plus pur. Ce sont les actions, et non pas l'apparence, qui témoignent de sa vraie nature. Si cela est vrai des religieux, cette vérité vaut également pour toute la société humaine.

\section{Amant, « vallet » de l'Antéchrist au même titre que Faux Semblant ?}

Le loup n'est pas seulement un symbole du clergé dans le Roman de la rose, mais représente toute action ayant pour but la satisfaction cruelle de ses désirs. Faux Semblant établit une comparaison entre l'Amant, qui doit attendre près de la tour de Jalousie où la Rose est enfermée, et le loup qui se cache à l'extérieur de la bergerie : «L'en ne voit pas tourjorz le leu, / Ainz prent bien ou tait les berbiz, / tout les gart l'en par les herbiz » ${ }^{38}$. Faux Semblant s'est déjà associé à Ysengrimus, le loup qui s'habille de la toison de Belin le bélier pour faire semblant d'être mouton. Tout comme l'hypocrite, le loup - la partie bestiale de l'homme - peut rester caché sous les apparences de la civilisation ou de l'humilité - «l'en ne voit pas tourjorz le leu »- et, en dépit de son comportement courtois dans le poème

\footnotetext{
${ }^{35}$ Ibid., v. 11049-11050.

${ }^{36}$ Ibid., v. 11087-11088.

${ }^{37}$ Ibid., v. 11061-11092.

${ }^{38}$ Ibid., v. 12468-12470.
} 
de Guillaume de Lorris, l'Amant est identifié, chez Jean de Meun, à la même rapacité bestiale et hypocrite que le frère le plus prédateur et le plus faux. Plus tard, Dangier confirmera cette identification quand il dira que laisser entrer Amant dans la tour de Jalousie avec Bel Accueil « ne seroit autre chose a fere / fors que par amoretes fines / metre Renart o les gelines $»^{39}$. Mettre le renard avec les poules semble analogue à mettre le loup avec les moutons et, dans les deux cas, les seules caractéristiques d'Amant sont son appétit vorace et son désir irrésistible de le satisfaire. Les paroles et le comportement courtois d'Amant sont révélés pour ce qu'ils sont : une fausse toison, et le personnage central du Roman de la rose est devenu moins un objet de pitié qu'une menace dont on devrait avoir peur. Grâce au personnage de Faux Semblant et à son assimilation des bêtes civilisées et parlantes que sont les hommes à des hypocrites qui « Antecrist attend $[$ ent $]{ }^{40}$, le faux amour lupin d'Amant serait même diabolique et assimilable aux crimes de l'Antéchrist et de ses «vallez», les hypocrites, au nombre desquels lui aussi compterait. Si ce jeune homme qui, à la fin du roman, cueille sa rose d'une manière si violente qu'on soupçonne plutôt un viol qu'un moment tendre de passion, avouait avec Faux Semblant être un « lou ravisable», il ne serait pas dans le faux. Faux Semblant vit, selon lui, le plus souvent chez les religieux car

je me vois hosteler

la ou je me cuit mieuz celer, s'est la celee plus seüre

souz la plus umble vesteüre.

Religieus sunt mout couvert, seculer sunt plus aouvert. ${ }^{41}$

Les religieux prétendent être purs et saints, mais, bien souvent les amants font de même. Si les religieux représentent le cas le plus extrême du désir

\footnotetext{
${ }^{39}$ Ibid., v. 12482-12484.

${ }^{40}$ Ibid., v. 11815.

${ }^{41}$ Ibid., v. 10981-10986.
} 
animal caché sous un extérieur civil ou saint, l'hypocrisie n'est pas leur apanage. Le mensonge et la fiction peuvent se trouver n'importe où, surtout chez les amants et les poètes. Bien qu'il soit faux de dire que la continuation de Jean de Meun est tout à fait pessimiste en ce qui concerne la nature humaine, on se tromperait gravement si l'on ne voyait dans le Roman de la rose que la fausse apparence, le faux semblant, d'une histoire de fin'amor élégante et courtoise. S'il faut se méfier des hypocrites religieux, on ne doit pas baisser sa garde contre les loups en vêtement d'amants et contre toute la littérature courtoise dont ils se servent pour séduire et leurs dames et leurs lecteurs.

\section{Le meurtre de Malebouche et le brouillage de la distinction entre l'homme et l'animal}

L'écart entre l'homme et l'animal ne semble donc plus être un abîme infranchissable et, dans le Roman de la rose, Faux Semblant lui-même ne dit pas que lui et les siens sont comme des loups, mais qu'ils sont ces loups : «dedanz somes lous ravisables » ${ }^{42}$. Cette affirmation est directement illustrée, dans la suite du roman, par le meurtre de Malebouche. Le meurtre lui-même est curieux. On pourrait à première vue penser que cette mise à mort allégorise le silence définitif imposé à la rumeur par l'utilisation de l'hypocrisie. L'amputation de la langue, qui suit le meurtre, irait en ce sens. Toutefois, ici comme ailleurs chez Jean de Meun, l'allégorie ne rend pas si aisément les armes ${ }^{43}$. Pour commencer,

\footnotetext{
${ }^{42}$ Nous soulignons.

${ }^{43}$ Voir l'article de Simon Gaunt sur la perturbation de l'allégorie et l'homosexualité dans la figure de Bel Accueil (Simon GAUNT, " Bel Accueil and Improper Allegory », New Medieval Literatures, Oxford, Clarendon Press, vol. 2, 1998, p. 65-93). Voir en particulier p. 69: "The text, far from inviting us to look through the literal to allegorical level of meaning, as if the literal were transparent, seems rather to take pleasure in banging our heads against the literal, as if it were hard and opaque surface. » (« Le texte, loin de nous inviter à regarder à travers le sens littéral pour atteindre le sens allégorique, comme si le sens littéral était transparent, semble plutôt prendre plaisir à
} 
l'hypocrisie ne peut réduire la médisance au silence que de manière provisoire, ce qui cadre mal avec l'arrêt définitif qu'implique la mort de Malebouche. Par ailleurs, une lecture allégorique simple ne rend pas compte de la présence inquiétante, aux côtés de Faux Semblant, d'Abstinence Contrainte, non plus qu'elle n'explique la violence mise en œuvre. Une fois que Malebouche a reconnu l'autorité de Faux Semblant et autorisé l'entrée dans le château, sa mort n'est plus nécessaire à la guerre contre Jalousie. Le meurtre paraît donc gratuit, et semble avoir été commis uniquement en vue du plaisir qui en résulte. Thomas d'Aquin définit précisément ce type d'actes :

proprie loquendo, feritas uel saeuitia dicitur secundum quam aliquis in poenis inferendis non considerat aliquam culpas eius qui punitur, sed solum hoc quod delectatur in hominum cruciatu. Et sic patet quod continetur sub bestialitate, nam talis delectationon est humana, sed bestialis, proueniens uel ex mala consuetudine uel ex corruptione naturae, sicut et aliae huiusmodi bestiales affectiones ${ }^{44}$.

En tuant Malebouche, Faux Semblant se montre bestial, il agit comme un loup : il me semble que dans la miniature du manuscrit Douce 195 de la Bodleian Library ${ }^{45}$, sa robe grise, sa position à quatre pattes plutôt que debout, et le fait qu'il tue Malebouche de ses mains, et non en utilisant un outil humain, soulignent le caractère lupin et prédateur du meurtre. La strangulation - «a .II. poinz l'estraint, si l'estrangle $»^{46}-$ a, de ce point de

venir nous cogner la tête sur le sens littéral, comme s'il s'agissait d'une surface opaque et dure $»$, nous traduisons).

44 «À proprement parler, brutalité ou sauvagerie s'appliquent à celui qui, lorsqu'il inflige une punition, ne prend pas en considération la culpabilité de celui qui est puni, mais seulement le plaisir qu'il peut prendre à torturer un homme. En conséquence, il est évident que cela relève de la bestialité : car un tel plaisir n'est pas humain, mais bestial, et vient soit d'une mauvaise habitude, soit d'une nature corrompue, de la même manière que les autres passions bestiales » (THOMAS D'AQUIN, Summa theologiae, éd. cit., IIa, IIae, q. 159, a. 2 co., nous traduisons).

45 Oxford, Bodleian Library, ms. Douce 195, $\mathrm{f}^{\circ} 89 \mathrm{r}^{\circ}$. Voir Roman de la rose Digital Library, romandelarose.org/\#read;Douce195, page consultée le 3 mars 2012.

${ }^{46}$ Guillaume DE LoRRIS et Jean DE MEUn, Le Roman de la rose, éd. cit., v. 12335. 
vue, un statut ambigu, car en même temps qu'elle témoigne de la bestialité du meurtre, elle nécessite l'utilisation d'une caractéristique propre à l'homme, les pouces opposables. Dans cette scène tout particulièrement, les frontières entre humains et animaux commencent donc à devenir légèrement floues : pour interpréter ce fait, il nous faut donc nous tourner à présent vers la science du XIII ${ }^{\mathrm{e}}$ siècle, pour voir comment les différences entre les espèces étaient comprises au temps de Jean de Meun.

Cette différence est rendue claire par Thomas d'Aquin, qui réaffirme que les animaux non humains ne peuvent avoir de raison. Le même besoin de préserver la supériorité humaine informe la discussion sur le statut des Pygmées dans le De Animalibus d'Albert le Grand ${ }^{47}$. Pour lui, Pygmées et singes possèdent une forme de prudence et d'instinct naturel très proche des pouvoirs de la raison ${ }^{48}$, mais ne vivent pas dans la société civilisée que la nature humaine exige et «si qui autem homines sunt siluestres, sicut Pygmeus, non secundum unam et eandem rationem nobiscum sunt dicti homines, sed habent aliquid hominis in quadam deliberatione et loquela $»{ }^{49}$. Sans la civilisation et la ratio de la loi et de la société, il serait impossible de distinguer l'homme de l'animal. Mais, à un autre moment du $D e$ Animalibus, dans un développement sur les hommes, se produit un changement d'accent significatif. Albert décrit en effet un processus similaire à celui évoqué par saint Augustin, et à sa suite Alexandre de Halès, lorsqu'il considère que les races monstrueuses sont effectivement

\footnotetext{
${ }^{47}$ Le De Animalibus d'Albert le Grand n'est sans doute pas le seul ouvrage à avoir discuté du cas des Pygmées au XIII ${ }^{\mathrm{e}}$ siècle, mais dans le milieu de l'Université de Paris, où il enseignait et où Jean de Meun a écrit sa continuation du Roman de la rose, il a dû être très influent.

48 «Pygmeus, qui non sequitur rationem loquelae, sed naturae instinctum» (« Les Pygmées, qui ne suivent pas le langage de la raison, mais plutôt l'instinct de nature », Albert le Grand, De animalibus libri XXVI, éd. cit., t. 1, I. 1. 3. 46, nous traduisons)

49 « Si, en conséquence, il y a des hommes qui sont sauvages, comme les Pygmées, ils ne sont pas appelés humains dans le même sens que nous, mais il y a quelque chose d'humain en eux, par un type de pensée et par la parole» (Ibid., I. 1. 3. 44, nous traduisons).
} 
susceptibles de descendre d'Adam et des fils de Noé, et que leur difformité est une punition divine pour leurs péchés ${ }^{50}$. Il écrit : «si aliquando aliquis hominum per electionem se mundo inferiorem fecerit, iam quasi honore humanitatis exutus, proprietatem accipit bestiae, et per concupiscentiam porcus, per iram canis, per rapinam leo, et sic de aliis dicitur fieri $»^{51}$. L'homme prend de nouveau "la nature d'une bête », comme dans le cas des Pygmées, mais par l'effet d'un libre choix («per electionem »), et non plus à la suite d'un manque de civilisation. Il devient alors une sorte d'animal hybride revêtant les pires aspects de différentes créatures. Albert poursuit en affirmant que les actions du corps peuvent alors corrompre l'âme et que « inemendabiles dicuntur inuerecundi, quia in aliquo mutati sunt a rationis honore pecorinae naturae assumentes irrationabilitatem $»^{52}$. Ce n'est alors plus la forme extérieure du pécheur qui change, mais plutôt

50 SaInt Augustin, Sancti Aurelii Augustini De Civitate Dei libri XXII, Berhard DOMBART et Alfons KALB (éd.), Stuttgart, Teubner, «Bibliotheca scriptorum

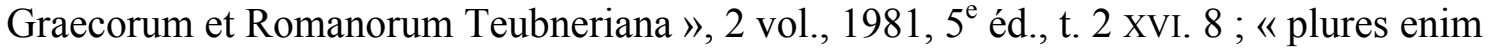
sunt rationes quare congruit corpora creaturarum rationalium esse monstruosa, qua non congruunt irrationalibus. Una est deordinatio peccati: solummodo enim inter res corporales homo deordinat se per peccatum, quod non faciunt bruta animalia; propter quod de divina iustitia est quod in poena deordinationis et deformationis culpae deordinationem et deformationem incurrunt in corpore " ( Car il y a plusieurs raisons qui peuvent expliquer que le corps des créatures rationnelles devienne monstrueux, des raisons qui ne peuvent pas s'appliquer aux créatures irrationnelles. L'une de ces raisons est le désordre créé par le péché ; car parmi les êtres corporels, l'homme est le seul qui puisse sortir de l'état qui lui a été attribué, et c'est une chose que les simples animaux ne peuvent pas faire. Pour cette raison, c'est par l'effet de la justice divine qu'en punition de la déviation et de la difformité de leur faute, ils encourent la déviation et la difformité du corps ", Alexandre DE HALÈs, Doctoris irrefragabilis Alexandri de Hales Ordinis minorum Summa Theologica, Bonaventura MARRANI (éd.), Quaracchi, Collegium S. Bonaventurae, 4 vol., 1924-1948, t. 2, II. 4. 2. 2. 1. 1. 3, ad. 5, p. 576, nous traduisons).

${ }^{51}$ « Si un homme choisit de se rabaisser lui-même en dessous de l'état civilisé, c'est comme s'il s'était privé lui-même de la dignité humaine, il prend les caractéristiques d'une bête, et on dit qu'il devient un cochon à cause de sa concupiscence, un chien à cause de sa colère, un lion à cause de ses rapines, et de même pour tous les autres vices » (AlBERT LE GRAND, De animalibus libri XXVI, éd. cit., t. 2, XXII, 1. 5. 9, nous traduisons).

52 «On dit que les pécheurs incorrigibles sont dépourvus de toute honte, car ils ont d'une certaine façon perdu la dignité de la raison, ayant pris la nature irrationnelle des bêtes » (Ibid., XXII, 1. 5. 11, nous traduisons). 
sa nature intérieure : alors que le pécheur semble le même extérieurement, la dégradation et la corruption prennent place intérieurement. Tout ce qui rend l'homme supérieur ou différent, c'est-à-dire la ratio ou raison, est effacé par les actions pécheresses, par la transgression des codes moraux, c'est-à-dire par des actes qui sont à la fois contraires à la raison et à la nature humaine. Alors que, dans le cas des Pygmées, Albert parvient, en évitant judicieusement de leur appliquer le terme de «monstres", à préserver la différence entre hommes et animaux, la dégradation par le péché réintroduit une combinaison gênante, contre-nature et potentiellement monstrueuse entre l'humain et l'animal, qui reste dissimulée sous la surface : une animalité rampante peut s'immiscer dans l'humain qui rejette le chemin de la vertu, c'est-à-dire de la raison. Or, c'est précisément ce qui se produit dans le cas de l'Amant, que Raison échoue à convaincre de la suivre. Amant lui-même affirme, à la fin du poème : «Mes de reson ne me souvint / Qui tant en moi gasta de peine $»^{53}$. Comme le pécheur décrit par Albert, l'Amant, par l'effet de la volonté, se métamorphose momentanément en animal incapable de faire usage de sa raison.

D'un autre côté, Albert, dans la discussion sur les Pygmées, reconnaît aux animaux l'usage d'une certaine forme d'intelligence. C'est également le cas pour le loup : « uades lupus per frondes lambit et lubricos facit pedes ne incessus audiatur $»^{54}$. Les animaux, et en particulier les loups, peuvent faire usage de stratagèmes qui, quoique rabattus sur l'imaginatio $^{55}$, ressemblent de manière alarmante à l'intelligence pratique

53 Guillaume DE LORRIS et Jean DE MEUn, Le Roman de la rose, éd. cit., V. 21730-21731.

54 «Quand un loup marche à travers des feuillages, il lèche ses pattes pour ne pas qu'on l'entende arriver » (ALBERT LE GRAND, De animalibus libri XXVI, éd. cit., t. 2, XXII, 2. 1. 114 , nous traduisons).

${ }^{55}$ La redécouverte du De motu animalium d'Aristote en Europe de l'Ouest a posé de sérieux problèmes aux commentateurs de la deuxième moitié du XIII ${ }^{\mathrm{e}}$ siècle: 
des hommes utilisée pour satisfaire les pulsions sexuelles. Nous avons vu que Faux Semblant assimile l'Amant au loup qui utilise l'astuce pour entrer dans la bergerie. De même, Ami - ainsi que tout amant -, dans sa hâte de satisfaire ses désirs charnels par n'importe quel moyen, dans son usage de la seule intelligence pratique à l'exclusion de la raison théorique, se met à ressembler de près à un loup poursuivant sa proie. L'apparence extérieure est humaine ; intérieurement, chacun a le caractère d'un animal (proprietas bestae). Faux Semblant peut alors apparaître sur la scène du Roman de la rose pour introduire une disjonction explicite entre l'apparence extérieure et la réalité intérieure, en s'appuyant sur une comparaison explicite de l'homme avec les bêtes sauvages. La fusion monstrueuse de l'animalité rapace et de l'humanité rationnelle se produit chez le pécheur d'Albert le Grand et chez tous ceux qui s'abandonnent à la satisfaction de leurs désirs, en retenant juste assez de l'intelligence pratique humaine pour masquer leur nature prédatrice derrière une façade d'honnêteté. Le loup n'est pas seulement au couvent, comme le souligne Faux Semblant, mais est répandu dans toute la société, chez tous ceux qui renoncent à un usage proprement humain de la raison, son usage théorique, qui seul permet véritablement de distinguer l'humain de l'animal. À la fin du poème - alors que le narrateur est sur le point de s'éveiller - l'animalité intérieure a même englouti l'humanité apparente.

Le Roman de rose de Jean de Meun met donc en scène des humains qui portent en eux une nature animale, qui peut échapper à notre contrôle et

« animaux » et « intelligence » y sont employés dans la même proposition à plusieurs reprises. De telles phrases posaient nécessairement la question de savoir si les animaux étaient mus par la raison. Cela débouchait sur des problèmes théologiques, et une solution a le plus souvent été trouvée en accordant aux animaux l'imaginatio, qui correspond à la phantasia aristotélicienne. Sur ce point, voir Pieter DE LEEMANS et Matthew KLEMM, « Animals and Anthropology in Medieval Philosophy », dans Brigitte RESL (dir.), A Cultural History of Animals in the Medieval Age, vol. 2, Oxford, Berg, 2007, p. 153-177, en particulier p. 169-170. 
nous transformer métaphoriquement en animaux, et qui peut rendre notre comportement entièrement semblable à celui des bêtes, différencié seulement par le degré d'intelligence pratique mise en œuvre. Jean fait donc de la métamorphose un aspect central de notre existence, que seule la société « retret un petit». Quoique internes, ces métamorphoses sont plus effrayantes encore, du fait de leur invisibilité potentielle, représentée par Faux Semblant et son utilisation trompeuse des vêtements. 
